\title{
Sprachlichen Anregungsgehalt im Elternhaus fördern?
}

\section{Evaluation eines Elterntrainings}

\author{
Lena Sielemann¹@, Jelena Hollmann², Uta Quasthoff ${ }^{3}$ und Elke Wild ${ }^{1}$ \\ ${ }^{1}$ Universität Bielefeld \\ ${ }^{2}$ Regionale Schulberatungsstelle der Stadt Bielefeld \\ ${ }^{3}$ Technische Universität Dortmund
}

\begin{abstract}
Zusammenfassung: Da familiäre Diskurspraktiken für die Entwicklung kindlicher Argumentationskompetenz wichtig sind, wurde das Training „Eltern fördern durch Argumentieren (EfA)“ für Eltern mit Kindern Anfang der Sekundarstufe I entwickelt. Kernziele sind, das Bewusstsein für die Rolle informeller Sprachförderpotentiale der Familie zu stärken und Strategien zum Umgang mit Konflikten zu vermitteln. Zur Evaluation von EfA wurde eine quasi-experimentelle-Feldstudie mit Prä-Post-Follow-Up-Design und unbehandelter Vergleichsgruppe realisiert. Von 37 teilnehmenden Eltern wurden 17 in sieben, wöchentlich stattfindenden Sitzungen trainiert. Die Befunde zur Akzeptanz des Trainings und nachhaltigen Umsetzung der vermitteln Strategien sind positiv. Auch zeigen sich erwartungsgemäß Veränderungen in der Interventionsgruppe: die Sensibilität trainierter Eltern für ihre unterstützende Rolle und die Bedeutung des sprachlichen Anregungsgehalts stieg stärker, sie zeigten einen deutlichen Zuwachs erwerbsförderlicher Diskurspraktiken, berichteten einen stärkeren Rückgang familialer Konfliktgespräche und neigten weniger zu unterdrückend-bestrafenden Reaktionen auf kindliche Wut. Die ermittelten Effektstärken lassen es sinnvoll erscheinen, das Training für die Nutzung in der Praxis aufzubereiten.
\end{abstract}

Schlüsselwörter: Elterntraining, sprachlicher Anregungsgehalt, Eltern-Kind-Interaktion

\begin{abstract}
Supporting the Use of Stimulating Linguistical Content at Home: Evaluation of a Parental Training Program
Abstract: Discursive practices at home are important for the development of children's reasoning skills. This led to the development of the training program "Eltern fördern durch Argumentieren (EfA)" (Parents Provide Support Through Reasoning), which addresses parents of children in secondary school. The aim of EfA is to raise awareness for parental support and to practice discursive strategies for dealing with conflicts. For the program evaluation, we carried out a quasi-experimental field study with pre-post/follow-up tests with an untreated control group. A total of 37 parents participated, 17 of whom were trained in seven weekly sessions. The acceptance was high, and learned strategies were integrated sustainably. Compared with the control group, parents in the intervention group achieved significantly greater increases in all parameters: They were more aware of their supporting role, showed greater levels of behavior supporting reasoning in interactions, reported a greater decline of conflicts, and showed less repressive-punitive reactions to their child's anger. Because of high effect sizes, this training is suggested for implementation.
\end{abstract}

Keywords: parent training, familial linguistic stimulus content, parent-child interaction

Die Fähigkeit gut zu argumentieren, erleichtert eine vollumfängliche Teilhabe am gesellschaftlichen Leben. Sie ermöglicht es, eigene Anliegen sachlich und überzeugend durchzusetzen sowie sich ein eigenes Urteil zu gesellschaftlichen Fragen und Debatten zu bilden. Zudem sind argumentative Kompetenzen für gute schulische Leistungen in allen Kernfächern (Quasthoff et al., 2015) und damit für den Bildungserfolg von Kindern wichtig (Kindt, 1999; Vogt, 2002). Eine gute Argumentationsfähigkeit dürfte zudem dazu beitragen, dass im frühen und mittleren Jugendalter zunehmende, um Rechte und Pflichten in der Familie kreisende Konfliktgespräche konstruktiv ge- führt werden und deshalb nicht die Eltern-Kind-Beziehung belasten.

Mit dem Eintritt in die Grundschule sind wesentliche Aspekte des Spracherwerbs z. B. die grundlegende morphologisch-grammatische Entwicklung abgeschlossen (Tracy, 2008). Im Verlauf des Schulalters beginnt dann die Herausbildung komplexer Diskursfähigkeiten, wie das Argumentieren, welches sich über das gesamte Pflichtschulalter hinweg weiterentwickelt (Quasthoff, 2011). Die Fähigkeit, Argumentationen zu analysieren, zu bewerten und selber mündlich sowie schriftlich zu produzieren, stellt eine fächerübergreifende Schlüsselkompetenz dar. Sie wird entsprechend von der Kultusministerkonferenz 
(KMK, 2012) als zentrales Bildungsziel benannt, allerdings nicht systematisch in allen Fächern der Sekundarstufe I gefördert (Wild et al., 2012). Für den Erwerb und die Entwicklung der Argumentationskompetenz wird daher dem Elternhaus als Einflussfaktor eine wichtige Bedeutung zugesprochen (Quasthoff et al., 2015). Um die elterliche Rolle bei der Entwicklung der Argumentationskompetenz von Kindern im Alltag zu stärken, wurde im Rahmen des BMBF-geförderten Projektes „Die Rolle familialer Unterstützung beim Erwerb von Diskurs- und Schreibfähigkeiten in der Sekundarstufe I" (FunDuS) ${ }^{1}$ das Training „Eltern fördern durch Argumentieren“ (EfA) entwickelt (Quasthoff et al., 2016). EfA verbindet unseres Wissens nach erstmals linguistische und psychologische Erkenntnisse, welche auf eine Steigerung der kindlichen Argumentationskompetenz durch elterliche Einstellungen, Verhaltens- und Kommunikationsmuster abzielen.

In der vorliegenden Studie wird untersucht, ob EfA Eltern bezüglich ihres Unterstützungspotentials für den Erwerb von Argumentationskompetenz sensibilisieren, den sprachlichen Anregungsgehalt in der Kommunikation mit Kindern nachhaltig steigern und einen konstruktiven, argumentativen Umgang mit Eltern-Kind-Konflikten vermitteln kann.

\section{Die Familie als Ort der informellen Bildung}

Lernprozesse außerhalb von Bildungsinstitutionen finden in der Regel erfahrungsbasiert, situativ und eher beiläufig statt (Rauschenbach, 2007). Sie wirken sich dennoch nachweislich auf die Kompetenzentwicklung und den Bildungserfolg von Kindern aus (Lehrl, Ebert, Blaurock, Rossbach \& Weinert, 2020; Reiss, Sälzer, Schiepe-Tiska, Klieme \& Köller, 2016).

Zur Bedeutung der Familie für die psychosoziale Entwicklung von Kindern liegen zahlreiche Studien vor: Eine sichere Bindung, ein autoritativer Erziehungsstil und ein gutes Familienklima sowie anregende Interaktions-/Kulturpraktiken ermöglichen Kindern nachweislich kurz- und langfristig einen erfolgreichen Umgang mit normativen Entwicklungsaufgaben sowie kritischen Lebensereignissen (Hohm et al., 2017; Werner \& Smith, 1982). An diesen Faktoren ansetzende Familienbildungsprogramme und Elterntrainings erzielten insgesamt gute Evaluationsergebnisse (Beelmann \& Schulz, 2013; Weiss, Schmucker \& Lösel, 2015) und unterstreichen dabei, dass zunächst nicht bewusst reflektierte Sozialisations- und Interaktionsroutinen im Rahmen umschriebener Interventionen veränderbar sind. Empirisch ebenfalls gut belegt ist, dass die Familie als Lernumwelt spezifische Potentiale (z.B. Zeit für Wiederholung, Anpassung an individuelles Leis- tungsniveau) bietet. Deren gezielte Nutzung, beispielsweise in Form eines aktiven Schulengagements von Eltern (z.B. Unterstützung bei den Hausaufgaben) fördert die Lern- und Leistungsentwicklung von Kindern über die gesamte Schulzeit hinweg (zusammenfassend Wild \& Walper, 2020). Der Einfluss des Elternhauses im Bereich der schriftsprachlichen Entwicklung von Kindern ist ebenfalls vielfach nachgewiesen (Hurrelmann, 2004; McElvany, Becker \& Lüdtke, 2009). Die Rolle der Familie für die Entwicklung von Argumentationskompetenz ist jedoch noch nicht umfassend untersucht. Bisherige Studien zeigen, dass sich interindividuelle Unterschiede in der Argumentationskompetenz teilweise auf familiäre Ressourcen, wie das Familieneinkommen (Salahu-Din, Persky \& Miller, 2008) oder die Anzahl der Bücher im Elternhaus (Klieme, 2008) zurückführen lassen. Neben diesen familienstrukturellen Anregungsfaktoren ist zusätzlich anzunehmen, dass vor allem die Qualität des sprachlichen Anregungsgehalts in der Familie für die Entwicklung von Argumentationskompetenz bedeutsam ist (Quasthoff \& Kern, 2007; Quasthoff et al., 2016).

\section{Sprachlicher Anregungsgehalt}

Unter dem sprachlichen Anregungsgehalt werden nach Hollmann, Wild, Quasthoff, Krah und Otterpohl (2012) verschiedene Aspekte des Sprachverhaltens innerhalb einer Familie zusammengefasst. Einen hohen sprachlichen Anregungsgehalt bieten Eltern, die ihren Kindern in alltäglichen Aushandlungen mit Respekt und auf Augenhöhe begegnen, die Sichtweisen ihrer Kinder tolerieren und berücksichtigen sowie ihre Kommunikation reziprok gestalten. Begründungen erleichtern das Verständnis elterlicher Entscheidungen und erhöhen die Wahrscheinlichkeit, dass Werte, Normen und Regeln internalisiert werden. Familiäre Gespräche sind häufig affektiv stark aufgeladen, unter solchen Umständen können jedoch keine sachlichen (Konflikt-) Gespräche geführt werden. Dies ist erst möglich, wenn Emotionen angemessen reguliert wurden. Eltern, die ihren Kindern vermitteln, dass auch negative Gefühle in der Familie ausgedrückt werden dürfen, ermöglichen die Entwicklung der dafür notwendigen sozial-emotionalen Kompetenzen, außerdem werden Kinder für die verschiedenen Ebenen von Kommunikation sensibilisiert (Hollmann et al., 2012). Zusätzlich stellen Eltern ein Argumentationsvorbild dar (Lernen am Modell, Bandura, 1986). Von einem hohen sprachlichen Anregungsgehalt wird ausgegangen, wenn argumentative Aushandlungen von Konflikten auch in der Paarbeziehung vorgelebt werden, Eltern eigene Standpunkte begründen und auch untereinander fair und sachlich disku-

Das Projekt wurde durch das Bundesministerium für Bildung und Forschung (BMBF) finanziert. Förderkennzeichen: $01 \mathrm{GJ} 1207$ A/B 
tieren. Konversationsanalytische Untersuchungen (Domenech \& Krah, 2014; Heller \& Krah, 2015) von ElternKind-Aushandlungen weisen darauf hin, dass Kinder bessere argumentative Leistungen zeigen, wenn Eltern ihnen die Möglichkeiten bieten, das Argumentieren zu üben und ihre Sichtweisen in Konversationen berücksichtigen. Im Sinne dieser Befunde wird im Rahmen des EfA Trainings der sprachliche Anregungsgehalt in Familien fokussiert, Wissen darüber und dessen Bedeutsamkeit vermittelt.

\section{Interaktionsmuster Fördern und Unterstützen}

In der Sprachwissenschaft wurden informelle Gesprächsmuster qualitativ analysiert und danach unterschieden, inwiefern sie die Ausbildung von Argumentationskompetenz unterstützen können (Quasthoff \& Kern, 2007). Es wurden drei Muster herausgearbeitet (Quasthoff \& Krah, 2012): Fordern und Unterstützen, Raumlassen und Akzeptieren sowie Übergehen und Selberlösen. Das Muster Fordern und Unterstützen wird als besonders hilfreich für den Erwerb von Argumentationskompetenz erachtete. Es ist dadurch gekennzeichnet, dass Eltern interaktive „Zugzwänge“ nutzen, mit deren Hilfe sie ihre Kinder zu einem stärkeren argumentativen Verhalten anregen können. EfA vermittelt, dass das elterliche Kommunikationsverhalten unmittelbar das Kommunikationsverhalten des Kindes beeinflusst. Eltern können durch interessiertes Nachfragen ihr Kind auffordern, eigene Standpunkte zu begründen und Gegenargumente aufzuführen. Dabei sollte Kindern genug Zeit gelassen werden, um auf Nachfragen $\mathrm{zu}$ reagieren oder eigene Lösungsvorschläge $\mathrm{zu}$ entwickeln. Zugzwänge im Gespräch werden durch WFragen (Wieso? Warum? etc.) erzeugt, die dem Kind einen Gesprächsraum mit vielen Gesprächsanteilen eröffnen.

\section{Umgang mit Streitgesprächen}

Wie die Meta-Analyse von Laursen, Coy und Collins (1998) zeigt, sind Konflikte zwischen Eltern und Kindern in der frühen Adoleszenz (nach der dort vorgenommenen Einteilung 10-12 Jahre) besonders häufig, wobei die emotionale Konfliktintensität von der frühen zur mittleren Adoleszenz (nach der dort vorgenommenen Einteilung 13-16 Jahre) steigt. Das Konfliktgeschehen in der Familie ermöglicht, explorative und strittige Gespräche im familialen Alltag einzuüben. Gleichzeitig wird eine Neujustierung der Rollen innerhalb der Eltern-Kind-Beziehung gefördert. Die zunächst asymmetrische ElternKind-Beziehung in eine zunehmend symmetrische $\mathrm{zu}$ transformieren und dazu Rechte und Pflichten neu auszuhandeln, gilt als wichtige Entwicklungsaufgabe in der Familie (Youniss \& Smollar, 1985). Können die dabei thematisierten Interessenskonflikte konstruktiv aufgelöst werden, beinträchtigen sie nicht die Qualität der ElternKind-Beziehung, sondern befördern die kindliche Auto- nomieentwicklung (Hofer, 2006). Dafür ist allerdings entscheidend, dass von Kindern ausgedrückte positive sowie negative Emotionen von Eltern nicht ignoriert, unterdrückt oder bestraft werden. Vorliegenden Längsschnittstudien zufolge steht ein solches Verhalten der Ausbildung kindlicher Emotionsregulationsfähigkeiten entgegen (Chaplin, Cole \& Zahn-Waxler, 2005; Eisenberg, Cumberland \& Spinrad, 1998). Deshalb werden im Rahmen des EfA-Trainings spezifische Strategien zum Umgang mit den eigenen und kindlichen Emotionen vermittelt und die Eltern unterstützt, eine familiäre Verhandlungskultur zu etablieren und damit einen geeigneten Rahmen zu schaffen, in dem Konflikte argumentativ ausgetragen werden können. Die Verringerung familiärer Konflikte stellt ebenfalls ein zentrales Ziel des Trainings dar.

Zusammenfassend zielt das EfA-Training, welches sich an Eltern von Kindern in der Sekundarstufe I richtet, darauf ab, teilnehmenden Eltern ihre Bedeutung und die Relevanz des sprachlichen Anregungsgehaltes für die Entwicklung der kindlichen Argumentationskompetenz zu verdeutlichen, ihnen Strategien zur Förderung kindlicher Argumentationskompetenz zu vermitteln und ihren Blick auf die langfristigen Vorteile einer in der Familie gepflegten „Verhandlungskultur“ zu lenken.

Im vorliegenden Beitrag werden deskriptive Befunde zur Akzeptanz des EfA-Trainings und inferenzstatistische Analysen zu dessen Wirksamkeit vorgestellt. Beides ist wichtig, weil eine hohe Akzeptanz eine notwendige Bedingung für die Inanspruchnahme und damit auch für die fortlaufende Implementation einer Maßnahme darstellt (Buschmann \& Ritter, 2013). Es soll überprüft werden, wie Eltern das Training (subjektive Ergebnisqualität), die Methoden und Rahmenbedingungen sowie die behandelten Themen (subjektive Strukturqualität) beurteilen und ob sie die Inhalte des Trainings auch vier Monate später in ihrem Alltag anwenden (Nachhaltigkeit im Alltag). Zusätzlich sind unter dem Aspekt der Qualitätssicherung Belege für Trainingseffekte vorzulegen.

\section{Hypothesen}

Es wird angenommen, dass die teilnehmenden Eltern nach dem EfA-Training im Vergleich zu den nicht trainierten Eltern im Verlauf des betrachteten Zeitraums ein größeres Bewusstsein für die Relevanz alltäglicher Gespräche in der Familie entwickeln, ihre Überzeugung, das eigene Kind in seiner Argumentationskompetenz fördern zu können, deutlicher steigern, einen stärkeren Anstieg im sprachlichen Anregungsgehalt erzielen, das Gesprächsmuster Fordern und Unterstützen vermehrt anwenden, vergleichsweise stärker auf unterdrückend-bestrafende Reaktion auf kindliche Wutäußerungen ver- 
zichten und von einer deutlicheren Abnahme der familialen Konflikte berichten. zeitpunkt zudem eine Broschüre, in der wichtige Trainingsinhalte zusammengefasst wurden.

\section{Stichprobe}

\section{Methode}

\section{Design und Rekrutierung}

Um die genannten Trainingseffekte zu untersuchen, wurde eine quasi-experimentelle Feldstudie mit unbehandelter Vergleichsgruppe durchgeführt. Die Datenerhebungen erfolgten für beide Gruppen zeitgleich zu drei Zeitpunkten im Verlauf des zweiten Schulhalbjahres in der fünften Klasse. Die erste Erhebung erfolgte vor dem Training (Prä-Test, im Frühjahr), die zweite (Post-Test) unmittelbar nach dem Training, sieben Wochen später und die Follow-Up Erhebung wurde vier Monate später (d.h. 20 Wochen nach dem Prä-Test, im Sommer) durchgeführt ${ }^{2}$.

Für die Vergleichsgruppe wurden Eltern aus zwei kooperationsbereiten Gesamtschulen gewonnen. Für die Interventionsgruppe wurden Eltern von einer weiteren Gesamtschule rekrutiert. Auf diese Weise sollte ein etwaiger Informationsaustausch zwischen den Gruppen verhindert und mögliche Schultypeffekte kontrolliert werden. In den Klassen der 5. Jahrgangsstufe wurden über die Lehrkräfte Elternanschreiben weitergeleitet, in denen das Forschungsprojekt vorgestellt und um Mitwirkung gebeten wurde. Eltern in der Vergleichsgruppe wurde mitgeteilt, dass es um die Teilnahme an einer Längsschnittstudie geht.

Ein Teilnahmekriterium war neben der besuchten Schulstufe des Kindes (5. Klasse), ausreichende Deutschkenntnisse. Eltern mit Sprachproblemen hätten absehbar Schwierigkeiten gehabt, den Ausführungen und Instruktionen im Training zu folgen, die schriftlichen „Hausaufgaben" zu erledigen und sich an Kleingruppenarbeiten $\mathrm{zu}$ beteiligen, was zulasten des Gewinns aller teilnehmenden Eltern gehen könnte.

Allen Familien wurde für die Bearbeitung der drei Fragebögen eine gestaffelte Aufwandsentschädigung von je $50 €$ in Aussicht gestellt. Denn vorliegende Studien (Heinrichs, 2006) ließen erwarten, dass so insbesondere Familien mit niedrigem sozio-ökonomischen Status für das Training gewonnen und eine Teilnahme zu allen Messzeitpunkten sichergestellt werden kann. Eltern aus der Vergleichsgruppe erhielten nach dem letzten Mess-
In der Interventionsgruppegruppe wurden 20 Eltern unter Berücksichtigung ihrer terminlichen Präferenzen einer von vier EfA-Gruppen zugeordnet. Die an den anderen beiden Schulen rekrutierten 20 Eltern wurden der Vergleichsgruppe zugeordnet. Zwei Personen sagten die Teilnahme aus privaten Gründen (Umzug und Krankheit) unmittelbar vor Beginn der ersten Trainingssitzung ab. Eine Person brach das Training nach dem ersten Termin aus gesundheitlichen Gründen ab. In den folgenden Analysen werden nur die Daten der Eltern berücksichtigt, welche das gesamte Training durchliefen. Insgesamt nahmen 37 Eltern, darunter 33 Frauen, an der Untersuchung teil. 17 Eltern gehörten der Interventionsgruppe an und 20 Eltern der Vergleichsgruppe. Die teilnehmenden Eltern waren im Mittel $41.46(S D=6.17)$ Jahre alt, $16.7 \%$ der Eltern hatten ein Kind, 38.9\% zwei Kinder, 40.5\% drei Kinder und 2.7\% fünf Kinder. Die Kinder der 5. Klasse (51.4\% weiblich) waren im Mittel 10.62 Jahre alt $(S D=.55)$.

In den teilnehmenden Familien wurde zu $86.1 \%$ immer und zu 13.9\% häufig Deutsch gesprochen. Der größte Teil der Kinder lebte mit beiden leiblichen Elternteilen zusammen (62\%), knapp ein Drittel wuchs in einer Ein-Elternteilfamilie auf $(27 \%)$ und $11 \%$ in einer Patchworkfamilie. Mit Rückgriff auf die International Standard Classification of Education (ISCED; UNESCO Institute for Statistics, 2012) zeigte sich, dass die Stichprobe zu einem großen Teil aus Familien mit hohem Bildungsniveau bestand (hoch: 62.2\%; mittel: 35.1\%; niedrig: $2.7 \%$ ), was bei der Interpretation der Ergebnisse zu berücksichtigen ist. In allen genannten soziodemografischen Merkmalen ungleichsgruppe nicht statistisch bedeutsam.

\section{Beschreibung des Trainings}

EfA umfasst sieben wöchentliche 1,5 stündige Sitzungen und sieht eine Vermittlung der Inhalte in Kleingruppen (je 2-5 Elternteile) vor. Die standardisiert umzusetzenden Trainingssitzungen wurden von einem interdisziplinären Team aus zwei geschulten Trainierinnen durchgeführt. terschieden sich die Eltern der Interventions- und Ver-

\footnotetext{
Zusätzlich zur Datenerhebung via Fragebögen erfolgte sowohl für die Interventions- als auch die Vergleichsgruppe zum Zeitpunkt des Präsowie des Post-Tests eine videografische Aufnahme von Eltern-Kind-Interaktionen. Diese Daten sind jedoch nicht Teil des vorliegenden Artikels.
} 
Tabelle 1. Inhaltlicher Ablauf und Themenschwerpunkte des EfA-Trainings

\begin{tabular}{|c|c|}
\hline Themenschwerpunkt & Zweck / Ziel der Sitzung \\
\hline $\begin{array}{l}\text { (1) Ressourcenorientierung und Sensibilisierung für die Bedeutung } \\
\text { von Argumentation }\end{array}$ & $\begin{array}{l}\text { Klärung organisatorischer Fragen, erster Zugang zu Argumentationskom- } \\
\text { petenz, deren Relevanz für Schulerfolg und Potentiale des Elternhauses } \\
\text { beim Erwerb der Argumentationskompetenz }\end{array}$ \\
\hline (2) Perspektiven für wünschenswertes Verhalten entwickeln & Schulung erwerbsförderlichen Verhaltens: Wirkung von Zugzwängen \\
\hline $\begin{array}{l}\text { (3) Interaktion in Alltagssituationen I: Einüben des Musters Fordern } \\
\text { und Unterstützens }\end{array}$ & $\begin{array}{l}\text { Kennenlernen und Einüben des Interaktionsmusters Fordern und Unter- } \\
\text { stützen }\end{array}$ \\
\hline $\begin{array}{l}\text { (4) Interaktion in Alltagssituationen II: Erhöhung der diagnostischen } \\
\text { Kompetenz der Eltern }\end{array}$ & $\begin{array}{l}\text { Tipps zum Umgang mit unterschiedlich kompetenten Kindern und zur } \\
\text { optimalen Zeitnutzung mit Kindern }\end{array}$ \\
\hline $\begin{array}{l}\text { (5) Grundlagen lerntheoretischer Mechanismen und Förderung ange- } \\
\text { messenen Verhaltens }\end{array}$ & $\begin{array}{l}\text { Vermittlung lerntheoretischer Strategien zur Förderung angemessener } \\
\text { Verhaltensweisen des Kindes, z. B. Lernen am Modell, Verstärkung }\end{array}$ \\
\hline $\begin{array}{l}\text { (6) Schwierige argumentative Interaktionen: Wenn das Kind nun gar } \\
\text { nicht will! Warum ist ruhig bleiben so wichtig und wie ist es möglich? }\end{array}$ & $\begin{array}{l}\text { angemessene Reaktion in Streitsituationen, Strategien zur Unterstützung } \\
\text { des Kindes beim Umgang mit schwierigen Situationen }\end{array}$ \\
\hline (7) Abschlusssitzung & Zusammenfassung der wichtigsten Inhalte, Reflektion des Trainings \\
\hline
\end{tabular}

Die Sitzungen sehen eine Kombination aus kurzen InputPhasen und ausgedehnten, interaktiven Phasen vor. In den interaktiven Phasen werden mithilfe von Videos, Rollenspielen, Selbsterfahrungen und Hausaufgaben die zentralen Informationen und Strategien vermittelt, eingeübt und unter dem Gesichtspunkt der Übertragbarkeit in den Alltag reflektiert. Die Themenschwerpunkte der einzelnen Sitzungen sind in Tabelle 1 zusammengefasst.

\section{Messinstrumente}

\section{Subjektive Ergebnis- und Strukturqualität sowie Nachhaltigkeit im Alltag.}

Im Post-Fragebogen wurde die subjektive Ergebnisqualität anhand von drei Items (z. B. „Wie hilfreich war das Training für Sie selbst?") auf einer fünfstufigen Skala (1 = gar nicht, $5=s e h r$ ) erfasst. Außerdem wurde die Strukturqualität mit drei Items erfasst, welche die Zufriedenheit mit den Aufgaben und Übungen (,Wie zufrieden sind Sie rückblickend mit den Aufgaben und Übungen, die in dem Training durchgeführt wurden?"), den Rahmenbedingungen (,Wie zufrieden sind Sie rückblickend mit dem zeitlichen Umfang des Trainings [Anzahl der Treffen, Länge der Sitzungen]?") und den Themen (,Wie zufrieden sind Sie rückblickend mit den im Training behandelten Themen?" $)$ jeweils auf einer vierstufigen Skala $(1=$ wenig, $4=$ sehr) abfragten.

Im Rahmen des Trainings vermittelte Kompetenzen und Verhaltensweisen (siehe Tabelle 2) wurden auf einer vierstufigen Skala $(1=$ nie, $4=$ immer $)$ in Bezug darauf bewertet, ob sie zum Zeitpunkt des Follow-Ups in den Alltag integriert wurden.

Trainingseffekte. Alle weiteren Instrumente wurden sowohl im Prä- als auch im Post- und Follow-Up-Test eingesetzt. Sofern nicht anders angegeben, entsprechen alle aufgeführten Skalen einem vierstufigen Antwortformat $(1=$ stimmt gar nicht, $4=$ stimmt ganz genau $)$. Die interne Konsistenz der Skalen wird für jeden Messzeitpunkt mit Cronbachs $\alpha$ angegeben und schwankt für alle verwendeten Skalen zwischen .90 und .57 , wobei die Werte größtenteils als gut zu bewerten sind.

Die Einstellungen zur Relevanz alltäglicher sprachlicher Interaktionen wurde anhand einer Skala zur Bedeutung des Elternhauses beim Erlernen der Argumentationskompetenz mit fünf selbstentwickelten Items (z. B.: „Kinder lernen im Gespräch mit ihren Eltern, wie man argumentiert."; Prä: $\alpha=.88$; Post: $\alpha=.89$; Follow-Up: $\alpha=.87$ ) abgebildet.

Die Selbstwirksamkeitsüberzeugung der Eltern, die Ausbildung der kindlichen Kompetenzen im häuslichen Umfeld fördern zu können, wurde anhand einer Skala mit fünf Items erfasst, welche anlehnend an die Scale of Parent Efficacy for Helping the Child Succeed in School (Hoover-Dempsey, Bassler \& Brissie, 1992) konzipiert wurde (z.B.: „Ich habe eine klare Vorstellung, wie ich mein Kind unterstützen kann, damit es argumentieren lernt."; Prä: $\alpha=.85$ Post: $\alpha=.90$; Follow-Up: $\alpha=.87$ ).

Die Ausprägung des sprachlichen Anregungsgehaltes wurde anhand der revidierten Fassung des Fragebogens zur Erfassung des Anregungsgehaltes in Familienkonversationen von Hollmann et al. (2012) erfasst. Es wurde der Gesamtwert herangezogen, weil laut Hollmann et al. (2012) die Facetten „Reziprozität in intergenerationalen Austauschprozessen“, „Toleranz gegenüber abweichenden Positionen“, „Begründungen in der Eltern-Kind-Interaktion“, „Emotionale Offenheit der Familienmitglieder" sowie "Modellierung von argumentativen Aushandlungen in der Paarbeziehung", auf einen Gesamtfaktor laden (Prä: $\alpha=.89$; Post: $\alpha=.90$ Follow-Up: $\alpha=.67$ ).

Zentrale Aspekte des Musters Fordern und Unterstützen wurden auf einer Skala mit vier selbstentwickelten Items 
Tabelle 2. Im EfA-Training erworbene Kompetenzen und Verhaltensweisen, Bewertung zum Zeitpunkt des Follow-Ups

\begin{tabular}{lc}
\hline Kompetenzen & $M(S D)$ \\
\hline Ich fordere mein Kind auf, seine Meinungen zu erklären/begründen. & $3.18(.64)$ \\
Ich interessiere mich für das, was mein Kind sagt, indem ich Fragen stelle. & $3.47(.51)$ \\
Ich gehe darauf ein, was mein Kind sagt, indem ich ihm mögliche Gegenargumente aufzeige. & $3.18(.64)$ \\
Ich fordere mein Kind auf, selbst nach einer Lösung zu suchen. & $3.00(.61)$ \\
Wenn mein Kind noch nicht so gut begründen kann, stelle ich allgemeine Fragen, die es zum Begründen auffordern. & $3.24(.56)$ \\
Ich bin ein Vorbild für mein Kind und verhalte mich so, wie ich es mir von ihm wünsche. & $3.12(.60)$ \\
Regeln, die ausgehandelt werden können, diskutiere ich mit meinem Kind aus. & $3.18(.64)$ \\
Wenn mein Kind begründet wütend ist, sage ich ihm, dass ich das verstehe, und es völlig normal ist, wütend zu sein. & $3.29(.69)$ \\
Wenn mein Kind wütend ist, erzähle ich ihm wie ich mich fühle, wenn ich wütend bin. & $2.94(.90)$ \\
\hline
\end{tabular}

Anmerkungen: Wie häufig werden die erlernten Kompetenzen nach dem Follow Up angewandt ( $1=$ nie, $2=$ manchmal, $3=$ häufig, $4=$ =immer).

erfasst (z. B. „Ich lasse meinem Kind Zeit im Gespräch, sodass es seine Meinung ausführen kann"; Prä: $\alpha=.64$ Post: $\alpha=.57$; Follow-Up: $\alpha=.66$ ).

Die familiäre Konflikthäufigkeit wurde anhand einer Skala mit acht Items erfasst, welche anlehnend an die Skala „Konflikt“ des Forschungsinstruments zur Erfassung des Familienklimas (von Maurice, 2004) konzipiert wurde (z. B. „In unserer Familie gibt es viel Streit.“; Prä: $\alpha$ $=.89$; Post: $\alpha=.84$ Follow-Up: $\alpha=.88$ ).

Reaktionen auf kindliche Wut, welche in hoch konfliktären argumentativen Interaktionen häufig und intensiv auftreten können, wurden mit dem Fragebogen Emotions as a Child-Skala (EAC; Magai, 1996) gemessen. Der Fragebogen besteht aus zwei Skalen, wobei hier ausschließlich unterdrückend-bestrafender Reaktionen (z.B. „Ich sage meinem Kind, dass es böse ist.") anhand einer vierstufigen Skala ( 1 = fast nie, 4 = fast immer) erfasst wurden (9 Items; Prä: $\alpha=.73$; Post: $\alpha=.80$ Follow-Up: $\alpha=.61$ ).

\section{Voranalysen}

Zur Überprüfung von Gruppenunterschieden vor dem Training wurde eine multivariate Varianzanalyse mit allen abhängigen Variablen durchgeführt. Auf univariater Ebene zeigen sich nur für die elterliche Selbstwirksamkeitsüberzeugung $\left(F(1,35)=5.82, p=.02, \eta^{2}=.14\right)$ und den sprachlichen Anregungsgehalt $\left(F(1,35)=4.35, p=.04, \eta^{2}\right.$ $=.11$ ) signifikante Unterschiede. Diese sind in der Diskussion zu berücksichtigen.

Für alle Analysen kann das Vorliegen der Sphärizität angenommen werden (Mauchly's Test alle $p>.05$ ).

\section{Datenanalyse}

Die Einschätzungen zur subjektiven Qualität des Trainings werden deskriptiv beschrieben. Zur Überprüfung der Trainingseffekte wurde eine multivariate, zweifakto- rielle Varianzanalyse mit dem Messwiederholungsfaktor Messzeitpunkt (Prä-, Post-Test, Follow-Up) und dem Faktor Gruppe (Interventions-, Vergleichsgruppe) durchgeführt. Kurz- sowie langfristige Trainingseffekte wurden mithilfe einfacher linearer Kontraste analysiert. Für die Berechnung der Kontraste fand nachträglich eine Bonferroni-Holm-Korrektur der p-Werte statt (Gaetano, 2013), um dem Problem der $\alpha$-Fehler-Kumulation zu begegnen. Nach Bühner und Ziegler (2012) kann die Höhe des Trainingseffekts für die Interventionsgruppe durch den Zeit $\mathrm{x}$ Gruppe Interaktionseffekt beschrieben werden, dabei ist eine Effektstärke von $\eta^{2}>.01$ als kleiner, $\eta^{2}>.06$ als moderater und $\eta^{2}>.14$ als starker Effekt zu bewerten.

\section{Ergebnisse}

\section{Subjektive Ergebnis- und Strukturqualität sowie Nachhaltigkeit im Alltag}

$94 \%$ der Eltern bewerten das Training im Post-Test als ziemlich $(n=7)$ bzw. sehr $(n=9)$ hilfreich für sich selber $(M=4.48, S D=.62)$ und ziemlich $(n=8)$ bzw. sehr $(n=8)$ nützlich für ihre Kinder $(M=4.41, S D=.62)$. Zudem berichtet die Mehrheit ( $n=8$ ziemlich, $n=8 \mathrm{sehr}$ ), dass sie nach dem Training besser auf ihr Kind eingehen konnten $(M=4.41, S D=.62)$.

Alle teilnehmenden Eltern waren retrospektiv ziemlich $(n=8)$ bzw. sehr $(n=9)$ zufrieden mit den Aufgaben und Übungen $(M=3.53, S D=.51) .88 \%$ waren ziemlich $(n=4)$ bzw. sehr $(n=11)$ zufrieden mit den Rahmenbedingungen des Trainings $(M=3.53, S D=.72)$. Alle Eltern waren im Post-Test ziemlich $(n=5)$ bzw. sehr $(n=12)$ zufrieden mit den im Training behandelten Themen $(M=3.71, S D=$ .45). Die Eltern nutzen die im Training erworbenen Kenntnisse und Strategien nach eigener Einschätzung nach vier Monaten häufig bis immer (siehe Tabelle 2). 


\section{Trainingseffekte}

Auf multivariater Ebene konnte sowohl ein signifikanter Haupteffekt für den Faktor Zeit $(F(12,132)=4.34 p<.001$, $\left.\eta^{2}=.28\right)$ als auch ein signifikanter Zeit $x$ Gruppe Interaktionseffekt $\left(F(12,132)=5.61 p<.000, \eta^{2}=.34\right)$ festgestellt werden. Im Folgenden werden die Ergebnisse auf univariater Ebene berichtet (siehe Tabelle 3 für alle Mittelwerte). Für die Einstellungen zur Relevanz alltäglicher sprachlicher Interaktionen wurde ein signifikanter Haupteffekt für den Faktor Zeit $(F(2,70)=5.34, p=.007$, $\left.\eta^{2}=.13\right)$ sowie ein signifikanter Zeit $\mathrm{x}$ Gruppe Interaktionseffekt $\left(F(2,70)=8.74, p<.001, \eta^{2}=.20\right)$ gefunden. Die Änderung der Einstellung hin zur Erkenntnis der Bedeutsamkeit des Elternhauses für die Vermittlung von Argumentationskompetenz vom Prä-Test zu den beiden späteren Messzeitpunkten ist in der Trainingsgruppe jeweils signifikant größer als in der Vergleichsgruppe (Prävs. Post-Test: $F(1,35)=14.50, p=.002, \eta^{2}=.29$; Prä-Test vs. Follow-Up: $\left.F(1,35)=8.15, p=.007, \eta^{2}=.19\right)$.

Für die Selbstwirksamkeitsüberzeugung der Eltern, die Ausbildung der kindlichen Kompetenzen im häuslichen Umfeld fördern zu können, wurde ein signifikanter Haupteffekt für den Faktor Zeit $(F(2,70)=30.92, p<$ $\left..001, \eta^{2}=.47\right)$ sowie ein signifikanter Zeit x Gruppe Interaktionseffekt $\left(F(2,70)=14.91, p<.001, \eta^{2}=.30\right)$ gefunden. Die Steigerung der elterlichen Selbstwirksamkeitsüberzeugung vom Prä- $\mathrm{zu}$ den beiden späteren Messzeitpunkten, ist in der Trainingsgruppe jeweils signifikant größer als in der Vergleichsgruppe (Prä- vs. PostTest: $F(1,35)=4.27, p<.001, \eta^{2}=.37$; Prä-Test vs. FollowUp: $\left.F(1,35)=17.72, p<.001, \eta^{2}=.34\right)$.

Für den sprachlichen Anregungsgehalt in der Familie wurde ein signifikanter Haupteffekt für den Faktor Zeit $\left(F(2,70)=8.53, p<.001, \eta^{2}=.20\right)$ sowie ein signifikanter Zeit $x$ Gruppe Interaktionseffekt $(F(2,70)=4.90, p=.01$, $\left.\eta^{2}=.12\right)$ gefunden. Die Steigerung des sprachlichen Anregungsgehaltes vom Prä-Test zu den beiden späteren Messzeitpunkten ist in der Trainingsgruppe jeweils signifikant größer als in der Vergleichsgruppe (Prä- vs. PostTest: $F(1,35)=5.13, p=.03, \eta^{2}=.13$; Prä-Test vs. FollowUp: $\left.F(1,35)=6.87, p=.026, \eta^{2}=.16\right)$.

Für die Anwendung des Musters Fordern und Unterstützen wurde ein signifikanter Haupteffekt für den Faktor Zeit $\left(F(2,70)=7.53, p=.001, \eta^{2}=.18\right)$ sowie ein signifikanter Zeit x Gruppe Interaktionseffekt $(F(2,70)=$ 5.94, $\left.p=.004, \eta^{2}=.15\right)$ gefunden. Die Steigerung der Anwendung des Musters Fordern und Unterstützen vom Prä-Test zum Follow-Up ist in der Trainingsgruppe signifikant größer als in der Vergleichsgruppe (Prä-Test vs. Follow-Up: $\left.F(1,35)=9.5, p=.008, \eta^{2}=.21\right)$.

Der Haupteffekt für die Konflikthäufigkeit wurde nicht signifikant, wohl aber der Zeit x Gruppe Interaktionsef- fekt $\left(F(2,70)=9.18, p<.001, \eta^{2}=.21\right)$. Die Abnahme der Konflikthäufigkeit zwischen dem Prä-Test und den beiden späteren Messzeitpunkten ist in der Trainingsgruppe jeweils signifikant größer als in der Vergleichsgruppe (Prä- vs. Post-Test: $F(1,35)=8.60, p=.006, \eta^{2}=.20$; PräTest vs. Follow-Up: $\left.F(1,35)=18.67, p<.001, \eta^{2}=.35\right)$.

Für die unterdrückend-bestrafenden Reaktionen auf kindliche Emotionen wurde ein signifikanter Haupteffekt für den Faktor Zeit $\left(F(2,70)=6.55, p=.002, \eta^{2}=.16\right)$ sowie ein signifikanter Zeit $\mathrm{x}$ Gruppe Interaktionseffekt $\left(F(2,70)=4.74, p=.012, \eta^{2}=.12\right)$ gefunden. Die Abnahme unterdrückend-bestrafender Reaktionen vom Prä- zum Post-Test ist in der Trainingsgruppe signifikant größer als in der Vergleichsgruppe (Prä- vs. Post-Test: $F(1,35)=.77$, $\left.p=.028, \eta^{2}=.16\right)$.

\section{Diskussion}

In diesem Beitrag wurden erste Ergebnisse zur Akzeptanz und Wirkung des interdisziplinär von Sprachwissenschaftler_Innen und Psycholog_Innen konzipierten Trainings EfA vorgestellt, welches sich an Eltern richtet, die die Entwicklung der Argumentationskompetenz ihrer Kinder unterstützen möchten und Sicherheit benötigen, wie sie dies - auch unter Vorzeichen von Abgrenzungsbestrebungen ihrer Kinder- tun können. Damit setzt es an dem Mangel an Elternbildungsprogrammen für den Ausbau des (sprachlichen) Anregungsgehaltes in Familien an. Die vorliegende quasi-experimentelle Feldstudie mit Vergleichsgruppe, Prä-Post-Follow-Up-Messung und BlockRandomisierung kommt der Aufforderung von Lösel, Schmucker, Plankensteiner und Weiss (2006) nach, in der Evaluation von Elterntrainingsprogrammen systematisch und kontrolliert vorzugehen.

Die teilnehmenden Eltern waren mit dem EfA-Training in seinem Aufbau und seiner inhaltlichen Gestaltung sehr zufrieden, sodass von einer hohen Akzeptanz des Trainings auszugehen ist. Das Gelernte wurde von den trainierten Eltern nach eigenen Angaben auch nach 4 Monaten im Alltag umgesetzt, was für eine nachhaltige Wirkung spricht.

Mit Blick auf die erzielten Trainingseffekte ist entscheidend, dass sich nur in der Interventionsgruppe die angestrebten Einstellungs- und Verhaltensänderungen zeigen und diese auch praktisch bedeutsam sind. So bildeten trainierte Eltern im Vergleich zu den Eltern ohne Training eine stärkere Einsicht bezüglich der Bedeutung alltäglicher, sprachlicher Interaktionen für die kindliche Argumentationskompetenz aus und trauten sich eher zu, die Entwicklung der kindlichen Argumentationskompetenz zu unterstützen. Die trainierten Eltern berichteten 
förderlicheres Gesprächsverhalten als die Vergleichsgruppe: Eltern aus der Interventionsgruppe gaben an, dass die familiale Kommunikation nach dem Training ein höheres sprachliches Anregungsgehalt beinhaltet und sie häufiger das argumentationsanregendes Gesprächsverhalten Fordern und Unterstützen einsetzen. Umgekehrt verringerte sich nur in der Interventionsgruppe der Einsatz unterdrückend-bestrafender Reaktionen auf den Ausdruck kindlicher Wut und die Häufigkeit von ElternKind-Konflikten. Dabei bleibt offen, ob das Training die Konflikthäufigkeit reduziert oder ob Konflikte von den Eltern nicht mehr als solche bezeichnet und erlebt werden, weil diese seltener eskalieren und argumentativ geführte Streitgespräche als selbstverständlicher Teil einer gelebten Verhandlungskultur wahrgenommen werden.

\section{Limitationen und Ausblick}

Einschränkend ist anzumerken, dass aufgrund des Stichprobenumfangs differenziertere Betrachtungen (z. B. Schichteffekte) nicht möglich waren. Zudem wurde kein Wartekontrollgruppendesign bzw. Vergleichsgruppe mit alternativer Intervention umgesetzt. Da die Gruppen gezielt an unterschiedlichen Schulen rekrutiert wurden, können zudem Kompositionseffekte prinzipiell nicht ausgeschlossen werden. Die Vergleichbarkeit der beiden Gruppen in allen soziodemografischen Variablen stützt aber die Vermutung, dass sich die Zusammensetzung der Schüler- und Elternschaft an den drei kooperierenden Gesamtschulen nicht unterschied und die Ergebnisse hierdurch nicht beeinflusst sind. Aus pragmatischen Gründen und um Selbstselektionsprozesse aufgrund zu hoher Anforderungen an die Teilnehmenden zu vermeiden, wurden keine Selbstauskünfte von den Kindern erhoben. Es bleibt somit offen, ob kindliche Charakteristika wie die Intelligenz die Trainingswirksamkeit moderieren. Zusammenhänge zwischen Intelligenz und Argumentationskompetenz sind zwar signifikant, liegen aber nur im moderaten Bereich (Quasthoff et al. 2016). Replikationsstudien mit einer größeren Stichprobe, bei denen die Kompetenzentwicklung sowie Personenmerkmale der Kinder berücksichtigt werden, sind daher wünschenswert. Zusätzlich waren die initialen Selbstwirksamkeitsüberzeugungen der Eltern sowie der sprachliche Anregungsgehalt in der Vergleichsgruppe höher ausgeprägt als in der Interventionsgruppe. Allerdings sind Deckeneffekte, die den hypothesenkonform ausbleibenden Anstieg in der Vergleichsgruppe erklären könnten, angesichts der Werteverteilung unwahrscheinlich. Weiterführende Untersuchungen sollten sich mit der Einsatzmöglichkeit des EfATrainings für spezifische Zielgruppen (z.B. Migrationshintergrund) sowie als Präventionsmaßnahme und der 
damit verbundenen Frage nach der Wirksamkeit bei Eltern mit höheren Ausgangskompetenzen beschäftigen und untersuchen, ob Faktoren wie die (anfängliche) Konflikthäufigkeit in den Familien die Wirkung des EfA-Trainings moderieren.

Diese Studie berücksichtigt nur die von den teilnehmenden Eltern wahrgenommenen Veränderungen. Linguistische Analysen (Domenech, Krah \& Hollmann, 2017) der vor und nach dem Training per Video erhobenen Interaktionsdaten konnten jedoch zeigen, dass EfA zu beobachtbaren Veränderungen der elterlichen Verhaltensebene beitragen kann.

Verglichen mit den für familienbezogene Präventionsprogramme gefundenen, zumeist mittleren Effekten (z. B. für Erziehungsverhalten; Weiss et al., 2015; Nowak \& Heinrichs, 2008) zeigt das EfA-Training nicht nur ähnliche, sondern teilweise höhere Effektstärken.

Nach der theoriegeleiteten Entwicklung und dem empirischen Wirksamkeitsnachweis könnte deshalb für das EfA-Training eine Implementation in institutionalisierte Bildungsangebote angestrebt werden. Damit das Training im Sinne von Beelmann (2006) in vorhandene Versorgungssysteme eingebracht werden kann, gilt es zuvor das Training mitsamt der verwendeten Materialien so aufzubereiten, dass es auch von Fachkräften in der Praxis durchführbar ist. Zudem ist der Implementationsprozess wissenschaftlich $\mathrm{zu}$ begleiten, um eine qualitativ hochwertige Umsetzung zu gewährleisten (Fries \& Souvignier, 2009). Grundsteine dafür wurden bereits gelegt: Im Rahmen eines Nachfolgeprojektes werden Materialien für die Schulung pädagogischer Fachkräfte zur Durchführung des EfA-Trainings oder einzelner Bausteine daraus entwickelt ${ }^{3}$. Einige Eltern berichten bei dem Follow-Up-Test, dass sie ein erneutes Treffen der Gruppe zur Auffrischung bzw. zur Diskussion der Erfolge für sinnvoll gefunden hätten, sodass bei einer weiteren Trainingsmodifikation die Einführung von „booster sessions“ eingeplant werden sollte.

\section{Fazit}

Insgesamt kann mit EfA ein neuartiges und wirksames Elterntraining angeboten werden, welches den teilnehmenden Eltern die Relevanz des sprachlichen Anregungsgehaltes für die Entwicklung der kindlichen Argumentationskompetenz aufzeigt, ihnen im Sinne des sprachlichen Anregungsgehaltes erwerbsförderliche Gesprächsstrategien (Fordern und Unterstützen) vermittelt und ihren Blick auf die langfristigen Vorteile einer in der Familie gepflegten „Verhandlungskultur“ lenkt.

\section{Literatur}

Bandura, A. (1986). Social foundations of thought and action. A social cognitive theory (Prentice Hall series in social learning theory). Englewood Cliffs, N.J.: Prenctice Hall.

Beelmann, A. (2006). Wirksamkeit von Präventionsmaßnahmen bei Kindern und Jugendlichen. Zeitschrift für Klinische Psychologie und Psychotherapie, 35, 151-162. https://doi.org/10.1026/ 1616-3443.35.2.151

Beelmann, A. \& Schulz, L. (2013). Elternbildung und Erziehungsberatung. In S. Andresen, C. Hunner-Kreisel \& S. Fries (Hrsg.), Erziehung. Ein interdisziplinäres Handbuch (S. 85-91). Stuttgart: Metzler.

Bühner, M. \& Ziegler, M. (2012). Statistik für Psychologen und Sozialwissenschaftler (3. Aufl.). München: Pearson Studium.

Buschmann, A. \& Ritter, E. (2013). „Heidelberger Elterntraining zur frühen Sprachförderung" in der Praxis. Wie zufrieden sind die Eltern? Sprache Stimme Gehör, 37, 24-29. https://doi.org/10. 1055/s-0033-1334906

Chaplin, T. M., Cole, P. M. \& Zahn-Waxler, C. (2005). Parental socialization of emotion expression: Gender differences and relations to child adjustment. Emotion, 5, 80 -88. https://doi.org/ 10.1037/1528-3542.5.1.80

Domenech, M. \& Krah, A. (2014). What familial aspects matter? Investigating argumentative competences of learners at the beginning of secondary schooling in the light of family-based resources. Learning, Culture and Social Interaction, 3, 77-87. https://doi.org/10.1016/j.lcsi.2013.12.006

Domenech, M., Krah, A. \& Hollmann, J. (2017). Entwicklung und Förderung der Argumentationskompetenz in der Sekundarstufe I: Die Relevanz familiärer Ressourcen. Bildung und Erziehung, 70, 91 - 108. https://doi.org/10.7788/bue-2017-0108

Eisenberg, N., Cumberland, A. \& Spinrad, T. L. (1998). Parental socialization of emotion. Psychological Inquiry, 9, 241-273. https://doi.org/10.1207/s15327965pli0904_1

Fries, S. \& Souvignier, E. (2009). Training. In E. Wild \& J. Möller (Hrsg.), Pädagogische Psychologie (S. 405 - 428). Berlin, Heidelberg: Springer. https://doi.org/10.1007/978-3-540-88573-3_ 17

Fritzler, N. J., Schlamann, F. \& Wild, E. (2020). Förderung des sprachlichen Anregungsgehaltes in Familienkonversationen: Evaluation eines Train-the-Trainer-Konzeptes zur Durchführung eines Elterntrainings durch geschulte Fachkräfte, Manuskript in Vorbereitung.

Gaetano, J. (2013). Holm-Bonferroni Sequential Correction: An EXCEL Calculator. https://doi.org/10.13140/RG.2.1.4466.9927

Heinrichs, N. (2006). The effects of two different incentives on recruitment rates of families into a prevention program. The Journal of Primary Prevention, 27, 345-365. https://doi.org/10. 1007/s10935-006-0038-8

Heller, V. \& Krah, A. (2015). Wie Eltern und Kinder argumentieren. Interaktionsmuster im längsschnittlichen Vergleich. Mitteilungen des Deutschen Germanistenverbandes, 62, 5-20.

\footnotetext{
Dieses Ziel wird mit dem Projekt EfA-TT verfolgt (Fritzler, Schlamann und Wild, 2020) Unter folgendem Link erhalten interessierte Leser kostenfrei die Materialien des weiter entwickelten EfA-Trainings: https://www.uni-bielefeld.de/efa-tt/
} 
Hofer, M. (2006). Wie Jugendliche und Eltern ihre Beziehung verändern. In A. Ittel \& H. Merkens (Hrsg.), Kindheit und Jugend aktuell (Bd. 26, S. 9-27). Wiesbaden: VS Verlag für Sozialwissenschaften. https://doi.org/10.1007/978-3-531-90278-4_1

Hohm, E., Laucht, M., Zohsel, K., Schmidt, M. H., Esser, G., Brandeis, D. et al. (2017). Resilienz und Ressourcen im Verlauf der Entwicklung. Kindheit und Entwicklung, 26, 230-239. https://doi.org/10.1026/0942-5403/a000236

Hollmann, J., Wild, E., Quasthoff, U., Krah, A. \& Otterpohl, N. (2012). Der Erwerb sprachlicher Kompetenzen im Elternhaus Erste Befunde zur Güte eines Fragebogens zur Erfassung des Anregungsgehalts von Familienkonversationen. Unterrichtswissenschaft, 40, 47-63.

Hoover-Dempsey, K. V., Bassler, O. C. \& Brissie, J. S. (1992). Explorations in parent-school relations. The Journal of Educational Research, 85, 287-294. https://doi.org/10.1080/00220671. 1992.9941128

Hurrelmann, B. (2004). Sozialisation der Lesekompetenz. In U. Schiefele, C. Artelt, W. Schneider \& P. Stanat (Hrsg.), Struktur, Entwicklung und Förderung von Lesekompetenz (S. 37-60). Wiesbaden: VS Verlag für Sozialwissenschaften. https://doi. org/10.1007/978-3-322-81031-1_3

Kindt, W. (1999). Was sollte man in der Schule über Argumentationen lernen? Überlegungen aus der Sicht neuerer Argumentationsforschung. Der Deutschunterricht, 51(5), $26-36$.

Klieme, E. (Hrsg.). (2008). Unterricht und Kompetenzerwerb in Deutsch und Englisch. Ergebnisse der DESI-Studie. Weinheim: Beltz.

KMK. Ständige Konferenz der Kultusminister der Länder in der Bundesrepublik Deutschland. (2012). Bildungsstandards im Fach Deutsch für die Allgemeine Hochschulreife. (Beschluss der Kultusministerkonferenz vom 18.10.2012). Verfügbar unter: https://www.kmk.org/fileadmin/Dateien/veroeffentlichungen_ beschluesse/2012/2012_10_18-Bildungsstandards-DeutschAbi.pdf

Laursen, B., Coy, K. C. \& Collins, W. A. (1998). Reconsidering changes in parent-child conflict across adolescence: A metaanalysis. Child Development, 69, 817-832.

Lehrl, S., Ebert, S., Blaurock, S., Rossbach, H.-G. \& Weinert, S. (2020). Long-term and domain-specific relations between the early years home learning environment and students' academic outcomes in secondary school. School Effectiveness and School Improvement, 31, 102-124. https://doi.org/10.1080/09243453. 2019.1618346

Lösel, F., Schmucker, M., Plankensteiner, B. \& Weiss, M. (2006). Abschlussbericht: Bestandsaufnahme und Evaluation von Angeboten im Elternbildungsbereich. Erlangen - Nürnberg: Friedrich-Alexander-Universität.

Magai, C. (1996). Emotions as a child: adult version. Unpublished scale. Brooklyn: Long Island University.

McElvany, N., Becker, M. \& Lüdtke, O. (2009). Die Bedeutung familiärer Merkmale für Lesekompetenz, Wortschatz, Lesemotivation und Leseverhalten. Zeitschrift für Entwicklungspsychologie und Pädagogische Psychologie, 41, 121-131. https://doi. org/10.1026/0049-8637.41.3.121

Nowak, C. \& Heinrichs, N. (2008). A comprehensive meta-analysis of Triple P-Positive Parenting Program using hierarchical linear modeling: Effectiveness and moderating variables. Clinical Child and Family Psychology Review, 11, 114-144. https://doi. org/10.1007/s10567-008-0033-0

Quasthoff, U. (2011). Diskurs- und Textfähigkeiten: Kulturelle Ressourcen ihres Erwerbs. In L. Hoffmann, K. Leimbrink \& U. Quasthoff (Hrsg.), Die Matrix der menschlichen Entwicklung (Bd. 1, S. 210-251). Berlin: De Gruyter. https://doi.org/10. 1515/9783110259766.210
Quasthoff, U. \& Kern, F. (2007). Familiale Interaktionsmuster und kindliche Diskursfähigkeit: Mögliche Auswirkungen interaktiver Stile auf diskursive Praktiken und Kompetenzen bei Schulkindern. In H. Hausendorf (Hrsg.), Gespräch als Prozess. Linguistische Aspekte der Zeitlichkeit verbaler Interaktion (Studien zur deutschen Sprache, Bd. 37, S. 277 - 306). Tübingen: G. Narr.

Quasthoff, U. \& Krah, A. (2012). Familiale Kommunikation als Spracherwerbsressource: Das Beispiel argumentativer Kompetenzen. In E. Neuland (Hrsg.), Sprache der Generationen (S.115-132). Mannheim: Duden.

Quasthoff, U., Wild, E., Domenech, M., Hollmann, J., Kluger, C., Krah, A. et al. (2015). Mündliches und schriftliches Argumentieren in der Sekundarstufe I. Können Eltern ihre Kinder beim Erwerb dieser Schlüsselkompetenz unterstützen? In A. Redder, J. Naumann \& R. Tracy (Hrsg.), Forschungsinitiative Sprachdiagnostik und Sprachförderung - Ergebnisse (S. 207-231). Münster: Waxmann.

Quasthoff, U., Wild, E., Domenech, M., Hollmann, J., Krah, A., Kluger, C. et al. (2016). FUnDuS II. Unveröffentlichter inhaltlicher Abschlussbericht.

Rauschenbach, T. (2007). Im Schatten der formalen Bildung - Alltagsbildung als Schlüsselfrage der Zukunft. Diskurs Kindheitsund Jugendforschung, 2, 439-453.

Reiss, K., Sälzer, C., Schiepe-Tiska, A., Klieme, E. \& Köller, O. (Hrsg.). (2016). PISA 2015. Eine Studie zwischen Kontinuität und Innovation. Münster: Waxmann.

Salahu-Din, D., Persky, H. \& Miller, J. (2008). The nation's report card: Writing 2007. National assessment of educational progress at grades 8 and 12. National, state, and trial urban district results. NCES 2008-468. National Center for Education Statistics.

Tracy, R. (2008). Wie Kinder Sprachen lernen. Und wie wir sie dabei unterstützen können (2., überarbeitete Auflage). Tübingen: francke Verlag.

UNESCO Institute for Statistics. (2012). International standard classification of education. ISCED 2011. Montreal, Quebec: UNESCO Institute for Statistics. https://doi.org/10.15220/97892-9189-123-8-EN

Vogt, R. (2002). Im Deutschunterricht diskutieren. Zur Linguistik und Didaktik einer kommunikativen Praktik (Reihe Germanistische Linguistik, Bd. 228). Berlin: De Gruyter. Verfügbar unter: https://www.degruyter.com/document/doi/10.1515/97831109 40794/html

Von Maurice, J. (2004). Generationenübergreifende Interessenrelationen im Spiegel einer Theorie der Person-Umwelt-Passung. Dissertation. Universität Trier, Trier.

Weiss, M., Schmucker, M. \& Lösel, F. (2015). Meta-Analyse zur Wirkung familienbezogener Präventionsmaßnahmen in Deutschland. Zeitschrift für Klinische Psychologie und Psychotherapie, 44, 27-44. https://doi.org/10.1026/1616-3443/ a000298

Werner, E. E. \& Smith, R. S. (1982). Vulnerable but invincible. A longitudinal study of resilient children and youth. New York: McGraw-Hill.

Wild, E., Quasthoff, U., Hollmann, J., Otterpohl, N., Krah, A. \& Ohlhus, S. (2012). Die Rolle familialer Unterstützung beim Erwerb von Argumentationskompetenz in der Sekundarstufe I. Diskurs Kindheits- und Jugendforschung, 7, 101-112.

Wild, E. \& Walper, S. (2020). Familie. In E. Wild \& J. Möller (Hrsg.), Pädagogische Psychologie (S. 237-268). Berlin, Heidelberg: Springer.

Youniss, J. \& Smollar, J. (1985). Adolescent relations with mothers, fathers, and friends. Chicago: University of Chicago press. 


\section{Förderung}

Diese Forschung wurde durch das Bundesministerium für Bildung und Forschung (BMBF) finanziert, Förderkennzeichen: 01GJ 1207 A/B

Open Access-Veröffentlichung ermöglicht durch die Universität Bielefeld.

\section{ORCID}

Lena Sielemann

(D) https://orcid.org/0000-0001-8195-5287

\section{Lena Sielemann}

Fakultät für Psychologie und Sportwissenschaft Abteilung für Psychologie

Universität Bielefeld

Postfach 100131

33501 Bielefeld

lena.sielemann@uni-bielefeld.de 